\title{
Experimental Demonstration of Cognitive Provisioning and Alien Wavelength Monitoring in Multi-domain EON
}

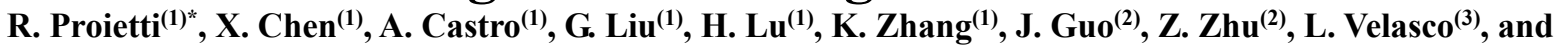 \\ S. J. B. Yoo ${ }^{(1)^{*}}$ \\ (1) University of California, Davis, CA 95616, USA; (2) University of Science and Technology of China (USTC), Hefei, China \\ (3) Universitat Politècnica de Catalunya (UPC), Barcelona, Spain \\ (*)rproietti@ucdavis.edu,sbyoo@ucdavis.edu
}

\begin{abstract}
This paper proposes a cognitive multi-domain EON architecture with machine-learning aided RMSA and alien wavelength monitoring. Testbed experiments show modulation format recognition, QoT monitoring and cognitive routing for a $160 \mathrm{GBd}$ alien multi-wavelength lightpath.

OCIS codes: (060.4251) Networks, assignment and routing algorithms; (060.1660); Coherent communications
\end{abstract}

\section{Introduction}

Elastic optical networking (EON) employs dynamic and adaptive lightpath provisioning for efficient and flexible network resources utilization. However, such flexibility and dynamicity demand for automated provisioning schemes with robust QoT estimation based on the actual network links conditions. Since analytical models offer rough estimates of the quality of transmission (QoT) of lightpaths [1], it is necessary to use high margins to compensate for model inaccuracies and guarantee the target QoT over the duration of the lightpaths. Such high margins reduce the maximum utilization of the network capacity. Therefore, more accurate situation-aware QoT estimations are desirable. To this aim, the authors in [2] proposed the use of machine learning (ML) techniques to estimate whether a given Route, Modulation format, and Spectrum Allocation (RMSA) would meet the desired QoT; their approach consisted in training a classifier using a large dataset with measures obtained over the time. Here, the classifier should be periodically re-trained to reduce deviations by constantly collecting and storing the monitored data. A different approach was presented in [3], where the authors proposed to continuously monitor the optical layer and use such data to tune an analytical impairments model used for QoT estimation. In [4], the authors demonstrated a single domain ML-based OSNR predictor in an SDN network. The scenario becomes more complex in multidomain environments, where lightpaths might not end in one single operator domain, but transparently traverse more of them; these lightpaths are known as alien wavelengths. Similarly as in [5], a broker can be used for end-to-end lightpath computation provided that QoT estimation can be performed in the broker. Note that such estimations must be done on a per-domain basis for accuracy and demarcation purposes, and hence, they require per-domain QoT alien wavelength monitoring. This paper proposes to add cognition to the broker by collecting per-domain QoT monitoring data to assist the end-to-end multi-domain RMSA process. The proposed cognitive multi-domain EON architecture makes use of a DNN machine-learning-aided RMSA and alien-wavelength monitoring. Monitoring is performed with a combination of a supervisory channel (SC) technique [6] and a novel modulation format identification (MFI) scheme [7] to discover the modulation format of alien signals.

\section{Architecture}

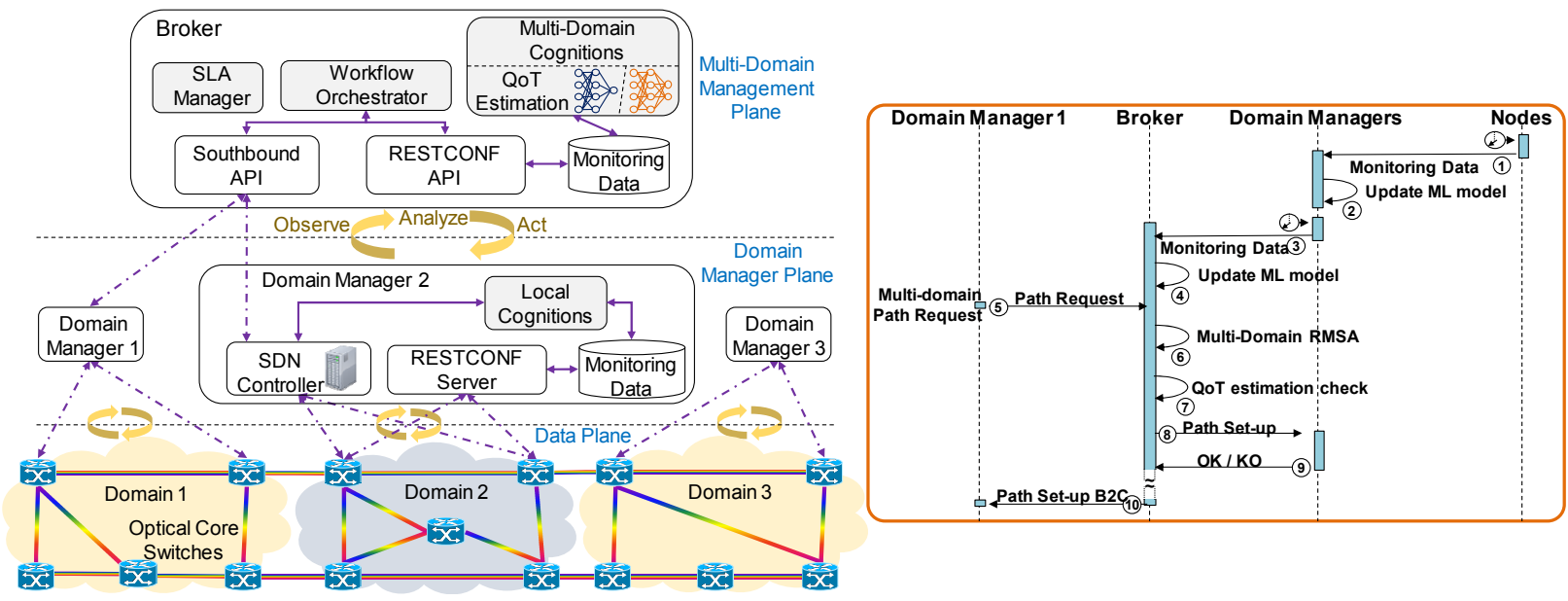

Fig. 1. (Left) A schematic framework of the proposed cognitive multi-domain optical network with observe-analyze-act machine learning cycle and the broker plane interacting with the multiple domain managers. (Right) Proposed workflow.

(C) 2018 Optical Society of America. Users may use, reuse, and build upon the article, or use the article for text or data mining, so long as such uses are for non-commercial purposes and appropriate attribution is maintained. All other rights are reserved.

https://doi.org/10.1364/OFC.2018.W4F.7 
Fig. 1 [left] depicts the proposed cognitive multi-domain EON architecture. Let us assume that each domain is managed by its own domain manager. On top of them, a broker coordinates end-to-end multi-domain provisioning. As in [5] the individual domains advertise to the broker their inter-domain links and an abstracted view of their topologies and spectrum availabilities (according to their particular SLA with the broker). The nodes are controlled by the SDN controller in the domain manager and they use the RESTCONF protocol to send monitoring data to the domain manager. The monitoring data is gathered in the local monitoring database and, in turn, it is periodically advertised to the broker. A YANG model was devised to transport the monitoring data collected by the nodes. The defined fields include the identifier of the node and the BER. The broker stores all the monitoring data received from the domain managers in the multi-domain monitoring database. Fig. 1 [right], illustrates the proposed workflow. Periodically, the nodes send the monitoring data collected from intra-domain and alien wavelengths connections, using the system shown in b, to the domain manager (step 1, Fig. 3d). The latter uses this information to update its machine learning algorithms' model (step 2). A similar process follows when the domain manager advertises its monitoring data to the broker (steps 3 and 4, Fig. 3d). When a new multi-domain request arrives at the broker (step 5), the latter executes its multi-domain RSMA (step 6). As explained in the introduction, this computation is done following a theoretical model, for this reason, the broker checks the solution obtained with its ML-aided QoT estimator (step 7). In case a solution is validated, the broker proceeds with the connection setup (steps 8, 9 and 10, Fig. 3d).

\section{ML-aided QoT Estimator for RMSA}

The ability to monitor the QoT of alien wavelengths enables the broker to obtain adequate information for learning the behavior of each domain, which in turn can facilitate the design of efficient multi-domain service provisioning algorithms. In this work, we present a DNN based QoT estimator to assist QoT-aware inter-domain RMSA operation (denoted as DNN-RMSA). Fig. 2 shows the principle of the proposed QoT estimator. The rationale behind using DNN is that with multiple hidden layer representations of the initial input data, we can potentially extract the most useful features for BER estimation when the inherent correlation within the data is sophisticated (considering a large-scale heterogeneous multi-domain network where limited intra-domain visibility is available).

For the proof-of-concept demonstration of our proposal, we implemented a QoT estimator with a DNN consisting of 5 layers $([6,10,20,10,1]$ activation function: $\mathrm{f}(\mathrm{x})=\max (\mathrm{x}, 0))$ considering the sample multi-domain topology in Fig. 2(a). We obtained the BER values of each inter-domain lightpath using a physical layer simulator which models the real transmission system e.g., taking into account the gain and power saturation of EDFAs and the links load. Evaluation over our testing data set indicates that the QoT estimator can achieve a prediction accuracy of $95.33 \%$.
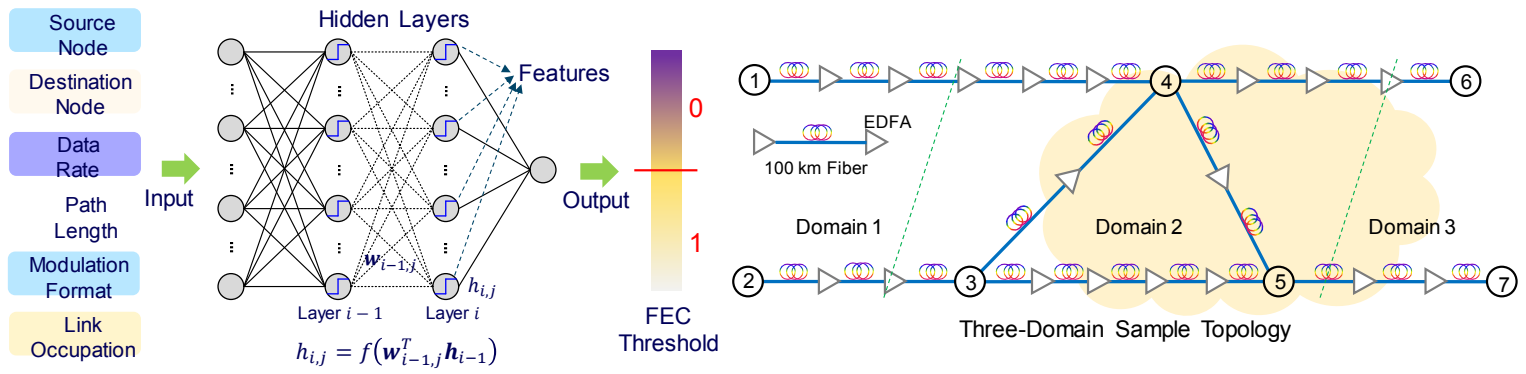

Fig. 2: (Left) Principle of DNN-based QoT estimator. The DNN in takes the source and destination nodes, data rate, physical length, modulation format and link occupation of an inter-domain lightpath candidate as input and outputs a flag indicating whether the BER of the lightpath satisfies the QoT requirement (i.e., below the FEC limit).

\section{Testbed Experiment}

The goal of the testbed demonstration is twofold. First, it shows a proof-of-concept demonstration of alien wavelength monitoring by a combination of MFI and SC techniques [5][6] (Fig. 3a). The information provided by the two monitoring functions can be jointly used by the middle DM to monitor the QoT of the alien signals. The QoT information could be then used by the DM and/or broker for training and updating the learning parameters of cognitive intra and inter-domain routing functions. Second, we show a scenario in which the use of a simple lookup table-based QoT could lead to underestimating the physical impairments (saturation and noise loading conditions in the optically-amplified links), resulting in a connection blocking due to insufficient BER at the Rx. Fig. 3b shows the experimental testbed. An I/Q modulator modulates $16 \mathrm{WDM}$ lasers at $10 \mathrm{GBd}$. An electrical arbitrary waveform generator (EAWG) produces the analog signals to generate QPSK or 8PSK alien wavelength signals. After EDFA amplification, the alien signals travel toward the middle domain. The ingress node has monitoring capabilities as MFI and QoT estimation based on SC technique. The ingress node applies a low-speed (200 Mb/s) low-modulationdepth SC on the alien channels to perform monitoring without significantly affecting the signals themselves. 


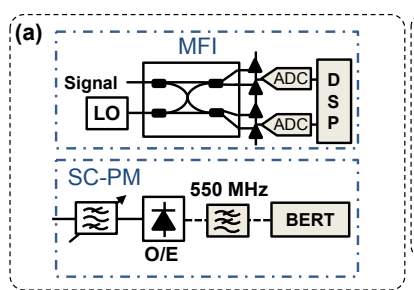

(b) DM Broker
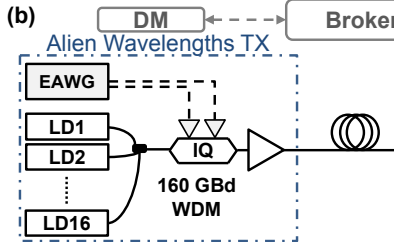

(c)

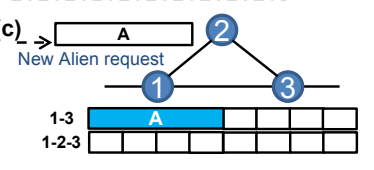

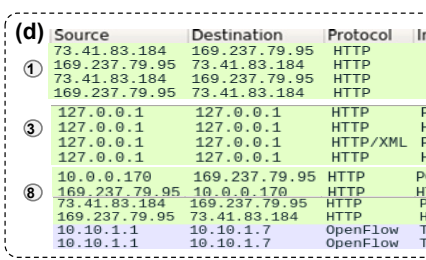
$\begin{array}{ll}16.10 .1 .1 & 70.16 \cdot 1.7 \\ 10.10 .1 .1 & 10.10 .1 .7\end{array}$
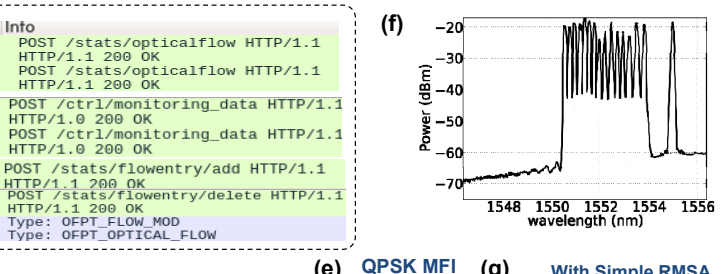

(e)

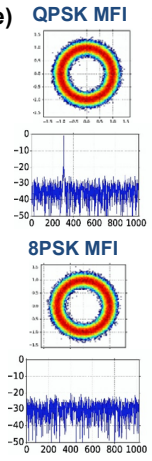

(g) With Simple RMSA
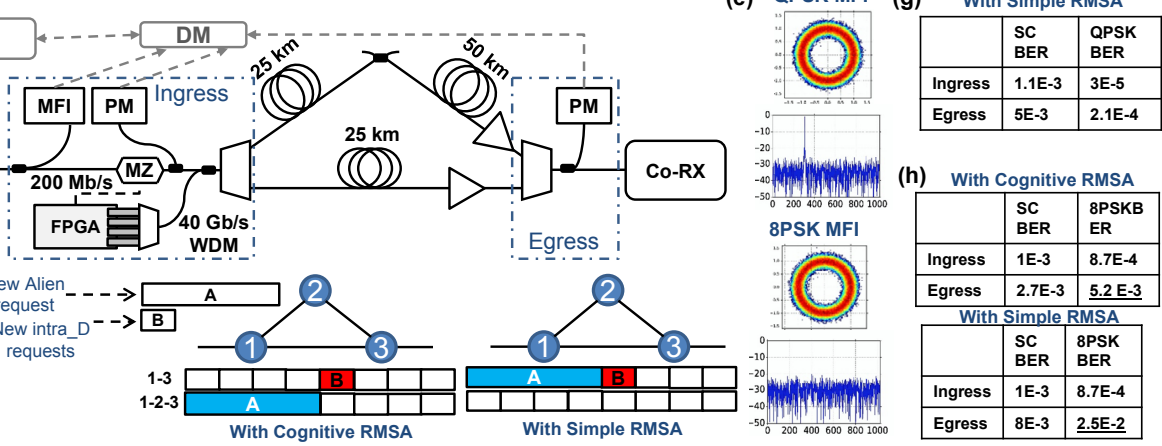

\begin{tabular}{l|l|l|}
\hline Egress & $5 \mathrm{E}-3$ & $2.1 \mathrm{E}-4$ \\
\hline
\end{tabular}

(h) With Cognitive RMSA

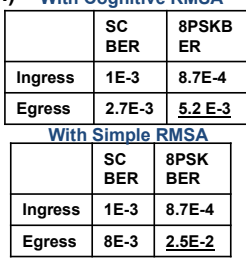

Fig. 3: (a) MFI monitoring and SC-PM monitoring: LO: local oscillator; ADC: analog to digital converter; DSP: digital signal processing; BERT: bit error rate tester; (b) Testbed. LD: laser diode; IQ: phase/quadrature modulator; MZ: Mach Zehnder modulator; DM: domain manager; (c) Left: scenario 1; Right: scenario 2 with and without cognitive RMSA; (d) Workflow Wireshark capture; (e) MFI output for QPSK and 8PSK signals, (f) Alien wavelengths spectrum; (g) monitor BER values for scenario 1; (h) monitor BER values for scenario 2.

Fig. 3a shows the schematic of MFI and SC-performance-monitoring blocks (please refer to [6],[7] for more details). At the ingress node, an FPGA with four WDM 10Gb/s TRXs acts as intra-domain traffic source. There are two paths to reach the egress node with length of $25 \mathrm{~km}$ and $75 \mathrm{~km}$, respectively. A PM block located at the egress node monitors the QoT of the alien signals going out of the domain. In scenario 1, the broker receives a $320 \mathrm{~Gb} / \mathrm{s}$ lightpath request $\mathrm{A}$. The broker decides to route the request through link 1-3 (shortest path - lightly loaded) of the middle domain using QPSK. The MFI block recognizes that the modulation format is QPSK based on the presence of a spectral tone after the 4th power operation and FFT function in its DSP block (see the difference with scenario 2 where for 8PSK there is not tone after the power operation and FFT block[7]).Then, by applying a SC and measuring its BER at the ingress and egress nodes, the DM can monitor the alien wavelengths' QoT by using preexisting correlation BER data stored in a database in the DM. Because the correlation data depends on the modulation format, it is essential to use the MFI block. In this case, the BER of the alien lightpath is below 20\%FEC threshold. In scenario 2, the broker receives a $480 \mathrm{~Gb} / \mathrm{s}$ lightpath request $\mathrm{A}$. In this scenario, there is also a WDM lightpath B (40 Gb/s) in link 1-3 already saturating the amplifier. In this case, the broker using a simple lookup table RMSA could decide to route the request through link 1-3 (shortest path) of the middle domain using 8PSK modulation format. However, because the link is already loaded by intra-domain traffic, the QoT estimation based on simple lookup table may lead to insufficient OSNR to guarantee proper QoT at the egress node (estimated BER is 2E-2 > FEC limit). Assuming instead that the broker has cognitive functions, the ML-aided QoT would take into consideration the current links status and decide to route the $420 \mathrm{~Gb} / \mathrm{s}$ alien signal through the longer but empty link 1-2-3. As shown by the BER tables in Fig. 3g, the BER at the egress node is now below the pre-FEC BER limit.

\section{Conclusions}

A cognitive broker-based multi-domain scenario has been demonstrated, where the broker estimates per-domain QoT using a DNN. Experiments include alien wavelength monitoring implemented by a combination of MFI and SC techniques.

This work was supported in part by DoE DEFC02-13ER26154, NSF CNS 1346688, and Spanish MINECO SYNERGY project (TEC201459995-R).

\section{References}

[1] P. Poggiolini, "The GN Model of Non-Linear Propagation in Uncompensated Coherent Optical Systems," IEEE/OSA JLT, 2012.

[2] L. Barletta et al., "QoT Estimation for Unestablished Lighpaths using Machine Learning," OFC, 2017.

[3] S. Oda et al., "A Learning Living Network with Open ROADMs," IEEE/OSA JLT, 2017.

[4] S. Yan et al., "Field trial of Machine-Learning-assisted and SDN-based Optical Network Planning with Network-Scale Monitoring Database," ECOC, 2017.

[5] A. Castro et al., "Brokered Orchestration for End-to-End Service Provisioning across Heterogeneous Multi-Operator (Multi-AS) Optical Networks," IEEE/OSA JLT, 2016.

[6] D. J. Geisler et al., "Experimental demonstration of flexible bandwidth networking with real-time impairment awareness," OSA OpEx, 2011.

[7] G. Liu et al., "Blind modulation format identification using nonlinear power transformation," submitted to OSA OpEx. 